\title{
Evolution of the Thrombolytic Treatment Window for Acute Ischemic Stroke
}

\author{
Andrew Stemer • Patrick Lyden
}

Published online: 20 January 2010

(C) The Author(s) 2010. This article is published with open access at Springerlink.com

\begin{abstract}
Ischemic stroke is a major cause of morbidity and mortality for which the only approved treatment in the acute setting is intravenous thrombolysis. The efficacy and safety of recombinant tissue plasminogen activator (rt-PA) have been firmly established within $3 \mathrm{~h}$ of symptom onset; however, few patients are eligible for treatment in this time window. Expanding the time for treatment has been challenging, but new evidence has demonstrated a modest statistical improvement in selected patients when rt-PA is administered within $4.5 \mathrm{~h}$. This important finding hopefully will enable more patients to receive treatment and simultaneously provides an opportunity to reaffirm that the benefits of rt-PA diminish with time.
\end{abstract}

Keywords Ischemic stroke · Intravenous rt-PA · ECASS III · Time window

\section{Introduction}

Despite extensive international research efforts, ischemic stroke remains the third leading cause of death and the most common cause of disability in industrialized nations [1]. Stroke affects more than half a million people in the United States every year [2] and will become more prevalent in an aging society increasingly afflicted with stroke risk factors, such as obesity, diabetes, and hypertension [3]. Although potentially debilitating, stroke also is the only neurologic disorder for which the symptoms may be completely reversed with appropriate treatment.

\footnotetext{
A. Stemer $(\triangle) \cdot$ P. Lyden

University of California,

San Diego, Medical Office North, Third Floor, Suite 3,

200 West Arbor Drive, \#8466,

San Diego, CA 92103, USA

e-mail: astemer@ucsd.edu
}

For many decades, a nihilistic approach toward stroke management led physicians away from actively treating stroke victims. This nihilism was unfortunate but understandable because no acute therapy was available to reverse the disease process. Thrombolytic medications such as streptokinase have been tested since the 1970s, but outcomes were consistently unsuccessful because of severe hemorrhagic complications [4]. In the 1980s, neurologists began investigating recombinant tissue plasminogen activator (rt-PA), and in a landmark article published in Science, Zivin et al. [5] demonstrated promising results using an embolic model. Further preclinical work established the relative safety of rt-PA for embolic stroke, although possible safety concerns with concomitant antiplatelet medications would require additional research [6].

The only approved therapy for acute ischemic stroke is rt-PA [7]. In large community surveys, its use has been frustratingly low; however, with organized stroke delivery teams, rates of $10 \%$ to $20 \%$ can be achieved $[8,9]$. There are many reasons patients do not receive acute treatment, but one major limitation is the rather short 3-hour time window used in the pivotal National Institute of Neurological Disorders and Stroke (NINDS) study [10]. Recently published randomized trials designed to expand this window may allow an increase in the number of eligible patients. We briefly review the historical background for the limited time window as well as recent studies that encourage an extension of the therapeutic time window for intravenous thrombolysis.

\section{Preliminary Studies}

One of the first randomized controlled trials (RCTs) testing the efficacy and safety of intravenous rt-PA in human subjects was the European Cooperative Acute Stroke Study (ECASS). This was a prospective, multicenter, double-blind, placebo-controlled clinical trial that randomly assigned 
patients to receive treatment with $1.1 \mathrm{mg} / \mathrm{kg}$ of intravenous rt-PA or placebo within $6 \mathrm{~h}$ from symptom onset. The primary outcome measures were the Barthel index and modified Rankin scale at 90 days. No significant differences between the groups were identified in the intention-to-treat analysis; furthermore, the rt-PA group suffered increased mortality and symptomatic intracranial hemorrhages (sICH). A target population analysis (removing protocol violations) favored rt-PA, but the trial ultimately was plagued by protocol violations and a poorly selected patient population [11]. When the subgroup of ECASS patients who were treated within $3 \mathrm{~h}$ was analyzed separately, however, a statistically significant benefit was found [12]. ECASS differed from the NINDS study in significant ways, including the important differences of using a higher dose, less strict blood pressure parameters, and an extended treatment window; these distinctions may have accounted for the lack of benefit and increased hemorrhage rate relative to the NINDS trial.

Learning from ECASS, the ECASS II trial was designed with a lower dose of rt-PA, also administered within $6 \mathrm{~h}$ of symptom onset. Stricter eligibility criteria included imaging and blood pressure guidelines, but again, rt-PA did not alter the primary end point. Similar to the first ECASS trial, ECASS II showed trends favoring rt-PA, but none was significant. sICH remained more common in the rt-PA group, although this difference did not lead to an overall increase in morbidity or mortality [13].

Simultaneously, another randomized, double-blind, multicenter, placebo-controlled clinical trial testing rt-PA was being conducted in the United States. Alteplase Thrombolysis for Acute Noninterventional Therapy in Ischemic Stroke (ATLANTIS) initially was designed to assess the efficacy and safety of intravenous rt-PA administered within $6 \mathrm{~h}$ of symptom onset, but safety concerns halted enrollment during the 5- to 6-hour window. The study before that point was renamed Part A, and going forward, new study patients enrolled between 0 and $5 \mathrm{~h}$ would constitute a separate trial (Part B). Eventually, ATLANTIS Part B was modified to a 3to 5-hour window after the NINDS results were published, but the study was stopped early because of slow recruitment. As with ECASS I and II, ATLANTIS could not support the use of rt-PA in patients treated 3 to $5 \mathrm{~h}$ after symptom onset. Again, rt-PA increased the rate of $\mathrm{sICH}$, but a comparison with the NINDS study suggested this was not caused by the increased treatment time.

\section{NINDS}

In 1995, the first successful randomized study using rt-PA in stroke was published. The NINDS study, like ECASS I/II and ATLANTIS, was a double-blind trial. Unlike prior studies, NINDS restricted the treatment time to less than $3 \mathrm{~h}$ from symptom onset and reduced the dose of rt-PA to $0.9 \mathrm{mg} / \mathrm{kg}$. Moreover, one half of the patients were treated within 90 min of symptom onset to maximize the likelihood of finding a benefit. NINDS consisted of two parts, the first measuring clinical activity within $24 \mathrm{~h}$ of stroke onset and the second using a global test to assess clinical outcome at 3 months.

In Part 1 , no statistically significant differences between groups were found using the primary outcome measure (improvement of $\geq 4$ points in National Institutes of Health Stroke Scale [NIHSS] score or complete resolution of neurologic deficits). It is worth remembering that the number of points (4) chosen to determine effectiveness was essentially a guess based on limited preclinical information, and post hoc analysis revealed statistically significant improvement using almost any other NIHSS improvement criteria or time strata [14]. All secondary outcome measures used in the first NINDS trial, including 3-month outcome on the modified Rankin scale and Barthel index, statistically significantly favored rt-PA treatment.

In contrast to the previous trials, NINDS Part 1 was the only study to use 24-hour improvement as a primary outcome measure. In retrospect, this could be a shortcoming of the study because it ultimately may not be a relevant assessment. Final neurologic recovery is most important, and this cannot be determined accurately at $24 \mathrm{~h}$. Although 3 months may not encompass the full extent of recovery either, it is much more reflective of eventual outcomes than the period when acute injury processes are still active.

Regardless of the debate surrounding 24-hour improvement, Part 2 of the NINDS trial assessed clinical results at 3 months as a primary outcome and did reveal statistically significant differences. Four primary outcomes were measured (Barthel index, modified Rankin scale, Glasgow outcome scale, and NIHSS), and the number of patients with favorable outcomes for each method was greater in the rt-PA group than in the placebo group. Compared with placebo, there was a $12 \%$ absolute increase and $32 \%$ relative increase in the number of patients with minimal or no disability among those treated with rt-PA. Importantly, this benefit was not offset with any increase in mortality. There were more symptomatic hemorrhages in rt-PAtreated patients $(6.4 \%$ vs $0.1 \%, P<0.001)$, but these patients had more severe baseline deficits and the proportion with hemorrhage was lower than in other RCTs with rt-PA [10].

The NINDS study demonstrated a clear benefit of rt-PA when used in the first $3 \mathrm{~h}$, and it was hoped that rt-PA would become widely used. Licensing bodies granted approval in the United States (1996), Canada (1999), and Europe (2002), but widespread adoption of thrombolytic therapy for acute stroke proceeded slowly as several challenges emerged. 
Initially, neurologists and emergency medicine physicians not experienced in making rapid decisions in stroke patients were reluctant to administer rt-PA [15]. Intravenous thrombolytic therapy represented a radical change from the previous standards of care for ischemic stroke that consisted largely of supportive therapy. rt-PA offered a high-risk/ high-reward alternative, but therapy often was withheld because of the fear of causing intracranial hemorrhage. Further analysis of hemorrhage rates from the NINDS data subsequently addressed many of these concerns by showing that the clinically relevant number needed to harm is close to 1 in 100 [16]. rt-PA now is fully accepted by almost all neurologists, but debate still exists among emergency medicine physicians, especially in areas where stroke specialists are not available to assist in the decisionmaking process [17].

At the same time, many patients are excluded from treatment because they are deemed to have neurologic deficits too mild or too rapidly improving to warrant the risks of rt-PA. Two published reports addressed this issue, and both found an unacceptably high number of patients (between one in three and one in four) deemed "too good to treat" on admission who were dead or dependant at discharge $[18,19]$. Decisions about thrombolytics in patients with mild symptoms are often difficult, but it should be remembered that patients with unstable disease processes can quickly improve before quickly declining. Such patients remain challenging, but current literature seems to support thrombolytic treatment [20].

\section{Extending the Time Window}

By far the largest obstacle restricting rt-PA in acute stroke treatment has been the time window [21]. Many patients do not arrive at the hospital within $3 \mathrm{~h}$, and even then, a comprehensive effort is required to evaluate a patient, make a treatment decision, and quickly administer rt-PA. Practically speaking, a patient must present to a hospital well before $3 \mathrm{~h}$ to have the opportunity to receive rt-PA. Individual cases require varying amounts of time to arrive at a treatment decision, but fulfilling clinical and diagnostic criteria generally takes about $60 \mathrm{~min}$ [8]. Up to $20 \%$ of stroke patients receive rt-PA at certified stroke centers $[8,9]$, but the overall percentage receiving treatment often is quoted at less than 5\% [22]. Extending the treatment window clearly would allow more patients to be treated; however, until recently the NINDS trial was the only successful rt-PA study, and a series of clinical trials already failed to broaden the window.

To explore a longer time window, a pooled analysis from six trials (NINDS Parts 1 and 2, ECASS I and II, and ATLANTIS Parts A and B) was performed. Despite minor differences in methodologies (ie, dose of rt-PA and time to treatment initiation), all studies had similar protocols, allowing the raw data to be pooled and analyzed, a more powerful technique than meta-analysis. Evaluating favorable 3-month outcomes (modified Rankin scale 0-1, Barthel index 95-100, and NIHSS 0-1) and occurrence of sICH were the main outcome measures.

As in both parts of the NINDS trial, the pooled analysis showed that the benefit of rt-PA diminishes as time elapses. A favorable outcome was most likely to occur in patients treated within $90 \mathrm{~min}$ and declined at every interval thereafter. Interestingly, the pooled analysis revealed that the odds ratio for a favorable outcome when treated between 3 and $4.5 \mathrm{~h}$ was 1.40 and the adjusted hazard ratio was not significantly worse at $4.5 \mathrm{~h}$ than $3 \mathrm{~h}$. These findings implied a beneficial effect of rt-PA beyond $3 \mathrm{~h}$ but, according to the same analysis, did not appear to extend to $6 \mathrm{~h}$ [23].

When the European Medicines Evaluation Agency (EMEA) approved rt-PA in 2002, it required two further studies. The first was to ensure that clinical application was as safe as that in study conditions, and the second was a trial extending the time window beyond $3 \mathrm{~h}$.

The Safe Implementation of Thrombolysis in StrokeMonitoring Study (SITS-MOST) was created to assess safety and efficacy by tracking sICH within $24 \mathrm{~h}$ and mortality at 3 months as primary outcomes. It became the largest stroke thrombolysis database and far exceeded the requirements of the regulatory authorities. The results showed that rt-PA in routine practice has a safety profile as good as or better than that seen in RCTs and is effective when used within $3 \mathrm{~h}$ of stroke onset [24].

The Safe Implementation of Thrombolysis in StrokeInternational Stroke Treatment Registry (SITS-ISTR) then used a post hoc analysis to compare 11,865 ischemic stroke patients treated with rt-PA under $3 \mathrm{~h}$ with 664 patients treated between 3 and $4.5 \mathrm{~h}$. No significant differences were found between the groups with regard to sICH, mortality, or independence at 90 days [25]. SITS-ISTR suggested the therapeutic window for safe, effective thrombolysis in acute stroke could be extended beyond $3 \mathrm{~h}$.

\section{ECASS III}

Despite the inability of previous RCTs to prove efficacy beyond $3 \mathrm{~h}$, SITS-ISTR suggested rt-PA was safe, and the pooled analysis suggested it was beneficial beyond established time windows. Further investigation was warranted, and by that time, the EMEA's second required study-the randomized trial with a time window beyond $3 \mathrm{~h}$-had begun.

ECASS III initially was created as a randomized, placebo-controlled, phase 3 trial testing the efficacy and 
safety of rt-PA administered 3 to $4 \mathrm{~h}$ after symptom onset. In 2005, based on results from the pooled analysis and slow recruitment, the study protocol was changed to allow an expanded time window from 3 to $4.5 \mathrm{~h}$.

ECASS III enrolled 821 patients from 19 European countries, 418 of whom received rt-PA; of these patients, $10 \%$ were treated between 3 and 3.5 h, $46.8 \%$ between 3.5 and $4 \mathrm{~h}$, and $39.2 \%$ between 4 and $4.5 \mathrm{~h}$. In the rt-PA group, $52.4 \%$ of patients achieved favorable outcomes, compared with $45.2 \%$ in the placebo group (absolute improvement of $7.2 \%, P=0.04$ ), and the global odds ratio resulted in better outcomes in patients treated with rt-PA. The sICH rate with rt-PA was higher than with placebo, but overall mortality was not affected. Comparing sICH among studies is challenging because of varying definitions, but regardless of the definition or expanded time window, the rate of sICH in ECASS III was not higher than previously reported.

ECASS III confirmed the NINDS finding of a significant benefit with rt-PA in the primary end point at 3 months. When rt-PA was given 3 to $4.5 \mathrm{~h}$ after the onset of stroke symptoms, a modest but significant improvement in clinical outcome was revealed. This earlier time to treatment is probably why ECASS III succeeded where previous, similarly designed trials did not. As discussed earlier, the effectiveness of rt-PA diminishes rapidly with time. Although ECASS I/II and ATLANTIS tested treatment windows between 3 and $6 \mathrm{~h}$, a disproportionate number of patients were enrolled just before time cutoffs. ATLANTIS Part B tested a treatment window from 3 to $5 \mathrm{~h}$ after symptom onset, but nearly $80 \%$ of patients were enrolled in the 4- to 5-hour interval and the average time to treatment for the entire trial was more than $4.5 \mathrm{~h}$. Knowing that rt-PA's benefit fades with time, it is not surprising, in retrospect, that previous trials did not succeed. In contrast, enrollment in ECASS III was evenly spread throughout the allowed time window; patients were not clumped near the end. In fact, the median time to enrollment was a reasonable $3 \mathrm{~h}$ and $59 \mathrm{~min}$. ECASS III also selected patients who may have been more likely to benefit from rt-PA. Although not supported by the NINDS findings, those with severe stroke (NIHSS $>25$ ) and a combination of prior stroke and diabetes were felt to be less likely to benefit and therefore were excluded from ECASS III [26•]. Finally, other trials simply did not contain sufficient sample size (power) to detect the modest $(7.2 \%)$ benefit seen in ECASS III.

\section{Conclusions}

ECASS III is an important step forward in treating stroke patients. Whereas physicians often were handcuffed by a strict 3-hour treatment window, now they have 90 extra minutes. Hopefully, more patients will receive therapy as a result, but based on the experience after the NINDS trial, time will be required before physicians feel comfortable with this change. A recent advisory paper from the American Heart Association/American Stroke Association (AHA/ASA) may help; it concluded that the pooled analysis and ECASS III data warranted a class I recommendation, level of evidence $\mathrm{B}$, that rt-PA should be offered between 3 and $4.5 \mathrm{~h}$ to select patients. Treating patients with ECASS III exclusion criteria, such as an NIHSS score greater than 25 , age greater than 80 years, and therapy with oral anticoagulation (regardless of international normalized ratio), is not established and, as such, received a class IIb recommendation, level of evidence C [27•].

ECASS III also serves to confirm the basic finding of the NINDS trial in an independent population: intravenous rt-PA treatment powerfully and safely ameliorates disability after acute ischemic stroke. Although mainstream neurologists and emergency medicine physicians accepted this finding long ago, residual skepticism remained in some quarters, fanned by irresponsible distortions from selfappointed "expert" naysayers. The replication of the NINDS trial in ECASS III should lay to rest all remaining skepticism about intravenous rt-PA for acute stroke.

Some of the most useful lessons from ECASS III ultimately may be what this study does not mean [28 $]$. The ideal treatment window is not anytime before $4.5 \mathrm{~h}$, and physicians cannot use the liberty of 90 extra minutes to delay treatment decisions. As the ECASS III authors astutely point out, early treatment remains critical, and the odds of a favorable outcome decline with every minute. In the NINDS study, for one patient to have a favorable outcome, the number needed to treat was 8.4 , but in the extended window of ECASS III, the number needed to treat rose to 14. Although benefit exists until $4.5 \mathrm{~h}$, the goal must be to keep the door-to-needle time as short as possible, generally within $1 \mathrm{~h}$.

Open Access This article is distributed under the terms of the Creative Commons Attribution Noncommercial License which permits any noncommercial use, distribution, and reproduction in any medium, provided the original author(s) and source are credited.

\section{References}

Papers of particular interest, published recently, have been highlighted as:

- Of importance

1. Thom T, Haase N, Rosamond W, et al.: Heart disease and stroke statistics-2006 update: a report from the American Heart Association Statistics Committee and Stroke Statistics Subcommittee. Circulation 2006, 113:e85-e151. 
2. Lloyd-Jones D, Adams R, Carnethon M, et al.: Heart disease and stroke statistics - 2009 update: a report from the American Heart Association Statistics Committee and Stroke Statistics Subcommittee. Circulation 2009, 119:480-486.

3. Ogden CL, Carroll MD, Curtin LR, et al.: Prevalence of overweight and obesity in the United States, 1999-2004. JAMA 2006, 295:1549-1555.

4. Thrombolytic therapy with streptokinase in acute ischemic stroke. The Multicenter Acute Stroke Trial-Europe Study Group. N Engl J Med 1996, 335:145.

5. Zivin JA, Fisher M, DeGirolami U, et al.: Tissue plasminogen activator reduces neurological damage after cerebral embolism. Science 1985, 230:1289.

6. Lyden PD, Zivin JA, Clark WA, et al.: Tissue plasminogen activator mediated thrombolysis of cerebral emboli and its effect on hemorrhagic infarction in rabbits. Neurology 1989, 39:703.

7. Lyden PD: Thrombolytic Therapy for Acute Stroke. Totowa, NJ: Humana Press; 2005.

8. Sattin JA, Olson SE, Liu L, et al.: An expedited code stroke protocol is feasible and safe. Stroke 2006, 37:2935-2939.

9. Grotta JC, Burgin WS, El-Mitwalli A, et al.: Intravenous tissuetype plasminogen activator therapy for ischemic stroke: Houston experience 1996 to 2000. Arch Neurol 2001, 58:2009-2013.

10. Tissue plasminogen activator for acute ischemic stroke. The National Institute of Neurological Disorders and Stroke rt-PA Stroke Study Group. N Engl J Med 1995, 333:1581.

11. Hacke W, Kaste M, Fieschi C, et al.: Intravenous thrombolysis with recombinant tissue plasminogen activator for acute hemispheric stroke. The European Cooperative Acute Stroke Study (ECASS). JAMA 1995, 274:1017-1025.

12. Steiner T, Bluhmki E, Kaste M, et al.: The ECASS 3-hour cohort. Cerebrovasc Dis 1998, 8:198.

13. Hacke W, Kaste M, Fieschi C, et al.: Randomised double-blind placebo-controlled trial of thrombolytic therapy with intravenous alteplase in acute ischaemic stroke (ECASS II). Lancet 1998, 352:1245.

14. Haley EC Jr, Lewandowski C, Tilley BC: Myths regarding the NINDS rt-PA Stroke Trial: setting the record straight. Ann Emerg Med 1997, 30:676.

15. Lyden PD, Lyden PD: Thrombolytic Therapy for Stroke. Totowa, NJ: Humana Press; 2001.

16. Saver JL: Hemorrhage after thrombolytic therapy for stroke: the clinically relevant number needed to harm. Stroke 2007, 38:2279-2283.
17. Hoffman JR, Schriger DL: A graphic reanalysis of the NINDS trial. Ann Emerg Med 2009, 54:329-336, 336.e321-336.e335.

18. Barber PA, Zhang J, Demchuk A, et al.: Why are stroke patients excluded from TPA therapy? Neurology 2001, 56:1015.

19. Smith EE, Abdullah AR, Petkovska I, et al.: Poor outcomes in patients who do not receive intravenous tissue plasminogen activator because of mild or improving ischemic stroke. Stroke 2005, 36:2497-2499.

20. Zivin JA: Acute stroke therapy with tissue plasminogen activator (TPA) since it was approved by the U.S. Food and Drug Administration (FDA). Ann Neurol 2009, 66:6-10.

21. Alberts MJ: TPA in acute ischemic stroke: United States experience and issues for the future. Neurology 1998, 51:S53.

22. Reeves MJ, Arora S, Broderick JP, et al.: Acute stroke care in the US: results from 4 pilot prototypes of the Paul Coverdell National Acute Stroke Registry. Stroke 2005, 36:1232-1240.

23. Hacke W, Donnan G, Fieschi C, et al.: Association of outcome with early stroke treatment: pooled analysis of ATLANTIS, ECASS, and NINDS rt-PA stroke trials. Lancet 2004, 363:768.

24. Wahlgren N, Ahmed N, Davalos A, et al.: Thrombolysis with alteplase for acute ischaemic stroke in the Safe Implementation of Thrombolysis in Stroke-Monitoring Study (SITS-MOST): an observational study. Lancet 2007, 369:275-282.

25. Wahlgren N, Ahmed N, Davalos A, et al.: Thrombolysis with alteplase 3-4.5 h after acute ischaemic stroke (SITS-ISTR): an observational study. Lancet 2008, 372:1303-1309.

26. - Hacke W, Kaste M, Bluhmki E, et al.: Thrombolysis with alteplase 3 to 4.5 hours after acute ischemic stroke. $N$ Engl J Med 2008, 359:1317-1329. This is a pivotal randomized controlled trial revealing statistically significant benefit when $r t-P A$ is administered between 3 and 4.5 hours after symptom onset.

27. - Del Zoppo GJ, Saver JL, Jauch EC, Adams HP Jr: Expansion of the time window for treatment of acute ischemic stroke with intravenous tissue plasminogen activator: a science advisory from the American Heart Association/American Stroke Association. Stroke 2009, 40:2945-2948. This is a recent advisory statement from the AHA/ASA regarding expansion of the time window to 4.5 hours as a class 1 recommendation in selected patients.

28. - Lyden P: Thrombolytic therapy for acute stroke - not a moment to lose. $N$ Engl J Med 2008, 359:1393-1395. This editorial about the ECASS III trial reiterates that minimizing time to treatment is the ultimate goal, regardless of an expanded time window. 